\title{
Avaliação funcional de pacientes com Acidente Vascular Cerebral utilizando o protocolo de Fugl-Meyer
}

\author{
Functional Evaluation of Patients patients with Stroke using the protocol of Fugl-Meyer \\ Mayara dos Santos Teles ${ }^{1}$, Cristiane Gusmão ${ }^{2}$
}

\begin{abstract}
RESUMO
Objetivo. O objetivo deste estudo foi avaliar a função motora dos pacientes com acidente vascular cerebral, e correlacionar idade e tempo de tratamento com o comprometimento motor através do protocolo de desempenho Físico de Fugl-Meyer. Método. Participaram 20 pacientes, com idade acima de 45 anos, sendo aplicado o protocolo de Fulg-meyer. Resultados. No estudo, 65\% eram do sexo masculino, com média de idade de 65,95 anos. A amplitude articular estava diminuída em $70 \%$ dos pacientes, 30\% apresentavam sensibilidade normal e $55 \%$ referiam alguma dor à movimentação passiva. Foi observado que $95 \%$ tinham diminuição da motricidade de membro superior e $40 \%$ no membro inferior. Os pacientes apresentaram grau de comprometimento motor severo (35\%) e marcante (65\%). Conclusáo. Não foi visto correlaçáo do grau de comprometimento motor com a idade e com o tempo de tratamento.
\end{abstract}

Unitermos. Reabilitação, Acidente Vascular Cerebral, Hemiplegia.

Citação. Teles MS, Gusmão C. Avaliação funcional de pacientes com Acidente Vascular Cerebral utilizando o protocolo de Fugl-Meyer.

\begin{abstract}
Objective. The objective of this study was to evaluate the motor function of patients with stroke and to correlate age and time of treatment with motor impairment through the Fugl-Meyer protocol of physical performance. Method. 20 patients aged over 45 years participated of this study in which Fugl-Meyer protocol was applied. Results. In this study $65 \%$ were male, with mean age of 65,95 years. Range of motion was decreased in $70 \%$ of patients, $30 \%$ presented regular sensibility and $55 \%$ reported pain on passive motion. It was observed that $95 \%$ had upper limb spasticity reduction and $40 \%$ had spasticity reduction at lower limbs. Patients presented severe (35\%) and outstanding (65\%) degree of motor impairment. Conclusion. It was not reported any correlation of motor impairment with age and time of treatment.
\end{abstract}

Keywords. Rehabilitation, Stroke, Hemiplegia.

Citation. Teles MS, Gusmão C. Functional Evaluation of Patients patients with Stroke using the protocol of Fugl-Meyer.
Trabalho realizado na Universidade Estadual do Sudoeste da Bahia, Campus Jequié, Jequié-BA, Brasil.

1.Acadêmica do Curso de Fisioterapia da Universidade Estadual do Sudoeste da Bahia, Feira de Santana-BA, Brasil.

2.Fisioterapeuta, Mestranda da Universidade Estadual do Sudoeste da Bahia, Vitória da Conquista-BA, Brasil.
Endereço para correspondência:

Cristiane Gusmão Rua Dalva Flores, n 60, apt 1502, Bairro Candeias CEP 45000-000, Vitória da Conquista-BA, Brasil. E-mail: cristianeaguiar@hotmail.com 


\section{INTRODUÇÃO}

O Acidente Vascular Cerebral (AVC) é definido como um sinal clínico de rápido desenvolvimento de perturbação focal da função cerebral com mais de 24 horas de duração, resulta da restrição na irrigação sanguínea ao cérebro, causando lesão celular e danos às funções neurológicas ${ }^{1}$. Sua incidência duplica a cada década de vida a partir dos 55 anos $^{2}$, no entanto, esse quadro vem mudando à medida que a presença de fatores predisponentes vem crescendo e cada vez mais um número maior de pessoas jovens cursa com essa afecção neurológica ${ }^{3}$.

A doença é uma das maiores causas de sequelas permanentes que geram incapacidades funcionais e representa a terceira causa de morte no mundo, perdendo apenas para o infarto agudo, e câncer ${ }^{4,5}$. É considerada a doença neurológica mais comum na prática clínica, a qual leva a déficits neurológicos do tipo paralisia total ou parcial do hemicorpo (hemiparesia e hemiplegia), além de comprometimentos no campo visual, sensorial e cognitivo ${ }^{6}$.

O AVC pode ser isquêmico ou hemorrágico. No primeiro, o tecido cerebral é privado do fornecimento de sangue arterial, em decorrência de um trombo ou embolo que obstrui o lúmen do vaso impedindo a passagem sanguínea. $\mathrm{O}$ hemorrágico resulta do extravasamento de sangue para fora dos vasos. O sangue pode extravasar para o interior do cérebro causando uma hemorragia intracerebral ou para um espaço entre o cérebro e a membrana aracnóide originando a hemorragia subaracnóidea ${ }^{1}$.

A hemiplegia contralateral ao lado da lesão encefálica é a disfunção motora prevalente nos casos de sequela de AVC. Além da deficiência motora também se observam distúrbios de sensibilidade, presença de espasticidade que ocorre depois de um período de hipotonia, perda dos padróes dos movimentos seletivos, alteração do equilíbrio, problemas de cognição entre outros distúrbios? ${ }^{7}$.

A espasticidade, acometimento comum causado pela lesão do sistema nervoso central é caracterizada por aumento da resistência ao alongamento muscular passivo, dependente da velocidade de estiramento do músculo, ocasionando hiperexcitabilidade dos reflexos miotáticos, hipertonia elástica, alteração da sensibilidade proprioceptiva, sendo, muitas vezes acompanhado por clônus, espasmos flexor e/ou extensor, contraturas, hiper-reflexia autonômica, distonia e por reflexos patológicos como Babinski ${ }^{8}$.

A patologia possui grande risco de deixar suas vítimas com incapacidades, de caráter temporário ou permanente, o que predispóe aos sobreviventes um padrão de vida sedentário com limitaçôes que implicam em necessidades variadas de grau de dependência, principalmente para o desenvolvimento das atividades básicas da vida diária9.

A reabilitação destes pacientes visa minimizar o impacto causado pelas alteraçôes da função sensóriomotora deixadas pelo AVC no sentido de promover independência funcional e melhorar a qualidade de vida dos mesmos. Desta forma, observa-se a importância de mensurar os resultados provocados na recuperação física bem como os fatores que interferem no programa de tratamento, desses indivíduos, a fim de direcionar a conduta adequada que propicie aos pacientes uma melhoria na assistência prestada.

Este estudo tem por objetivo avaliar a função sensório-motora dos pacientes com Acidente Vascular Cerebral em tratamento, bem como analisar se há correlação entre a idade, o tempo de tratamento com o comprometimento motor destes indivíduos, através do Protocolo de Desempenho Físico de Fugl-Meyer, buscando direcionar melhor as condutas para promover independência funcional e qualidade de vida aos pacientes.

\section{MÉTODO}

\section{Amostra}

Trata-se de um estudo descritivo analítico de corte transversal. Os locais do estudo foram a Clínica Escola de Fisioterapia da UESB e o Núcleo de Municipal de Prevenção e Reabilitação Física de Jequié Dr. José Maria Magalhães Netto (NUPREJ), situados em Jequié-BA, e estes foram escolhidos por concentrar o maior número de pessoas com sequelas de Acidente Vascular Cerebral (AVC) do município.

Compondo uma amostra probabilística e casual simples, participaram da pesquisa 20 indivíduos, acima de 45 anos, ambos os sexos, que se encontrava em tratamento fisioterapêutico, no período proposto para a coleta 
de dados, com diagnóstico Clínico de Acidente Vascular Cerebral. Foram adotados como critérios de inclusão: possuir sequela de AVC (déficit sensório-motor); estar em reabilitação fisioterápica, no mínimo por dois meses, independente do sexo; aceitação espontânea para participar da pesquisa, firmando o Termo de Consentimento Livre e Esclarecido, segundo a Resolução 196/96 do Conselho Nacional de Saúde (protocolo no 110/2009). Foram adotados como critérios de exclusão da pesquisa indivíduos que apresentassem déficits de compreensão, que impedissem o entendimento do protocolo.

\section{Procedimento}

$\mathrm{O}$ instrumento de coleta de dados utilizado foi o protocolo de avaliação da função sensório-motora da recuperação do AVC, Fugl-Meyer (EFM), que avalia seis aspectos do paciente: a amplitude de movimento, dor, sensibilidade, funçáo motora da extremidade superior e inferior e equilíbrio, além da coordenação e velocidade. A pontuação máxima da extremidade superior é igual a sessenta e seis pontos (66) e a da inferior, trinta e quatro (34), dando uma pontuação da função motora máxima igual a cem pontos (100), o que define a função motora normal. Foi estabelecido ainda quatro níveis de comprometimento motor: nível severo corresponde a pontuaçôes menores que 50 , que representam pequena ou nenhuma movimentação voluntária dos membros afetados; nível marcante com pontuação de 51 a 84; nível moderado com pontuação entre 85 e 95, onde especificamente a função da mão pode estar altamente comprometida e nível leve de comprometimento motor quando a pontuação ocorre de 96 a 99. O equilíbrio é avaliado por sete itens, utilizando-se a mesma escala de graduação. A sensibilidade é testada através da exterocepção e propriocepção recebendo uma classificação de (0) para anestesia, (1) hipoestesia e (2) sensibilidade normal. A movimentação passiva é avaliada com pontuaçóes de (0) para nenhum ou quase nenhum grau de movimentação; (1) perda de alguns graus de movimentação e (2) para amplitude normal de movimento. Para a avaliação da dor, (0) significa dor pronunciada, (1) alguma dor e (2) ausência de dor.

Os questionários foram aplicados individualmente sob forma de entrevista por um único pesquisador com o intuito de evitar erros de interpretação.

\section{Análise Estatística}

Os resultados foram expressos em frequências e médias e o método estatístico realizado para associação das variáveis foi o teste Exato de Ficher considerando significante $\mathrm{p}<0,05$, sendo que o programa utilizado foi SPSS versão 13.0.

\section{RESULTADOS}

\section{Dados demográficos}

Dos 20 pacientes avaliados $65 \%$ eram do sexo masculino e $35 \%$ do sexo feminino (Tabela 1), com média de idade de 65,95 variando entre 46 a 84 anos. Em relação ao estado civil, $50 \%$ dos pacientes eram casados, sendo $30 \%$ solteiros e $20 \%$ viúvos.

O lado mais afetado pelo AVC foi o direito, correspondendo uma porcentagem de $60 \%$ em relação ao lado esquerdo (40\%). A hipertensão arterial foi a patologia de maior distribuição nos pacientes (100\%), seguida da diabetes mellitus (15\%), colesterol (5\%) e arritmias (5\%). Foi observado que 65\% dos pacientes utilizavam algum dispositivo para ajudar na marcha. Em relaçáo ao tempo de tratamento, pode-se observar que a metade dos pacientes $(50 \%)$ tinha mais de um ano de reabilitação fisioterapêutica.

\section{Avaliaçáo sensório-motora}

Foi observado limitação de amplitude articular em $70 \%$ dos pacientes, sendo que $5 \%$ apresentavam apenas alguns graus de movimento e $25 \%$ com movimentação passiva normal, destes indivíduos 55\% cursavam com dor ao movimento passivo. Quanto a sensibilidade 30\% encontravam-se normais, já o restante $(70 \%)$ constatouse hiposinesia/disestesia, alteração proprioceptiva e exteroceptiva. Em nenhum paciente foi constatado equilíbrio normal, $15 \%$ não tinham equilíbrio e $85 \%$ tinham pelo menos alguma alteração de equilíbrio.

$\mathrm{Na}$ função motora de membro superior nenhum dos indivíduos avaliados conseguiram a pontuação máxima (66 pontos), 5\% receberam pontuação 0 e $95 \%$ cursavam com pontos abaixo do valor previsto, sendo as tarefas de retração (15\%) e abdução (20\%) e adução (45\%) de ombro menos realizadas. Já na função de membro inferior todos os membros da pesquisa tinham algum tipo de alteração, mas nenhum obteve valor mínimo e apenas 
$40 \%$ dos pacientes realizavam perfeitamente o exercício de dorsiflexão (Tabela 2).

Na limitação da amplitude de movimento (ADM) do membro superior foram observadas limitação principalmente de flexão (45\%) e abdução de ombro (50\%) e na supinação de braço (50\%). Já no membro inferior há limitação de $\mathrm{ADM}$ principalmente em dorsiflexão de tornozelo, onde apenas $40 \%$ dos pacientes apresentam grau de movimentação passiva normal, e na eversão do pé, 55\% (Tabela 1). Os pacientes sentem dores a movimentação passiva de membro superior a abdução de ombro e a flexão de cotovelo, não houve nenhum plano de movimento em que a dor se acentuasse em membro inferior.

Avaliado o comprometimento motor dos pacien- tes $35 \%$ apresentaram grau severo, $65 \%$ marcante e nenhum teve alteração motora moderada ou leve (Tabela 3). Foi feito uma associação entre idade, tempo de tratamento e comprometimento motor através do teste exato de Fisher. Não houve associação entre idade e o comprometimento motor, no qual o $p$-valor $=0,50$. Também pelo teste exato de Fisher não houve associação entre tempo de tratamento e grau de comprometimento motor, pois o $p$-valor $=0,342$.

\section{DISCUSSÃO}

Este estudo contou com participação de 20 pacientes com sequela de Acidente Vascular Cerebral dos quais mais da metade $(65 \%)$ pertenciam ao sexo masculino,

Tabela 1

Perfil dos pacientes avaliados na Clinica Escola de Fisioterapia e no Núcleo de Municipal de Prevenção e Reabilitação Física de Jequié Dr. José Maria Magalhães Netto (NUPREJ)

\begin{tabular}{|c|c|c|c|}
\hline \multicolumn{2}{|c|}{ Caracteristicas Gerais } & \multirow[b]{2}{*}{7} & \multirow{2}{*}{$\begin{array}{l}\% \\
65\end{array}$} \\
\hline Gênero & Feminino & & \\
\hline & Masculino & 13 & 35 \\
\hline & Total & 20 & 100 \\
\hline \multirow[t]{5}{*}{ Idade } & 45 a 55 anos & 2 & 10 \\
\hline & 55 a 65 anos & 8 & 40 \\
\hline & 65 a 75 anos & 7 & 35 \\
\hline & $>75$ anos & 3 & 15 \\
\hline & Total & 20 & 100 \\
\hline \multirow[t]{4}{*}{ Estado Civil } & Solteiro & 6 & 30 \\
\hline & Casado & 10 & 50 \\
\hline & Viúvo & 4 & 20 \\
\hline & Total & 20 & 100 \\
\hline \multirow[t]{4}{*}{ Tempo de Tratamento } & 2-6 meses & 8 & 40 \\
\hline & 6-12 meses & 2 & 10 \\
\hline & $>12$ meses & 10 & 50 \\
\hline & Total & 20 & 100 \\
\hline \multirow[t]{3}{*}{ Lado Acometido } & Direito & 12 & 60 \\
\hline & Esquerdo & 8 & 40 \\
\hline & Total & 20 & 100 \\
\hline \multirow[t]{5}{*}{ Patologias Associadas } & HAS & 20 & 100 \\
\hline & Diabetes & 3 & 15 \\
\hline & Colesterol & 1 & 5 \\
\hline & Arritmias & 1 & 5 \\
\hline & Total & 20 & 100 \\
\hline \multirow[t]{3}{*}{ Dispositivo para Marcha } & Sim & 13 & 65 \\
\hline & Não & 7 & 35 \\
\hline & Total & 20 & 100 \\
\hline
\end{tabular}


Tabela 2

Avaliação da função sensório-motora dos pacientes através do protocolo de desempenho Físico de Fugl-Meyer

\begin{tabular}{lccc}
\hline Avaliaçáo da Funçáo Sensorio-motora & \% Normal & \% Alguma Alteraçáo & \% funçáo Ausente \\
\hline Amplitude de Movimento (PM=44) & 25 & 70 & 5 \\
Dor (PM=44) & 45 & 55 & 0 \\
Funçáo Sensorial (PM=24) & 30 & 70 & 0 \\
Equilibrio (PM=14) & 0 & 85 & 15 \\
Função motora de membro superior (PM = 66) & 0 & 95 & 5 \\
Funçáo Motora de membro inferior (PM = 34) & 0 & 100 & 0 \\
\hline
\end{tabular}

$\mathrm{PM}=$ pontuação máxima

corroborando desta forma com a maioria dos achados da literatura que traçam o perfil de indivíduos que tiveram esta afecção neurológica ${ }^{10,11}$. Essa pode ser justificada pelo fato das mulheres estarem mais atentas e ter mais acesso ao serviço de saúde, reduzindo desta forma os riscos a desenvolver doenças ${ }^{12}$.

A média de idade dos pacientes 65,9 anos, e a maior quantidade de indivíduos tiveram faixa etária entre 60 e 80 anos. A idade é um fator de risco isolado para o AVC e cerca de $30 \%$ ocorre antes dos 65 anos, $70 \%$ nos indivíduos com 65 anos ou mais e o risco dobra a cada década em pacientes acima de 55 anos, principalmente devido a alteraçóes cardiovasculares e metabólicas ligadas à idade ${ }^{12}$. No entanto, alguns estudos já evidenciam a incidência em adultos jovens com esta afecção cerebrovascular, estando relacionados outros fatores de risco, tais como distúrbios de coagulação, doenças inflamatórias, imunológicas e uso de drogas, apesar destes não fazerem parte da grande maioria das pessoas com a doença ${ }^{3}$.

Em relação ao estado civil, metade dos indivíduos eram casados, assim como os resultados da pesquisa de pacientes com acidente vascular cerebral precoce, atendi-

Tabela 3

Nivel de Comprometimento Motor dos pacientes com Acidente Vascular Cerebral avaliados

\begin{tabular}{cccc}
\hline \multicolumn{4}{c}{ Comprometimento Motor } \\
\hline & Pontuaçáo & n & $\%$ \\
\hline Grave & $<50$ & 7 & 35 \\
Marcante & $51-84$ & 13 & 65 \\
Moderado & $85-95$ & ---- & --- \\
Leve & $96-99$ & $-\cdots--$ & --- \\
\hline
\end{tabular}

dos pelo sistema único de saúde, que analisou as incapacidades e identificou se há diferenças de gêneros em sobreviventes de primeiro episódio de AVC, em que também prevaleceu o estado civil casado ${ }^{13}$.

Em relação ao lado acometido, o direito foi mais frequente $(60 \%)$, contudo, a literatura mostra variaçōes nessa frequência a exemplo do estudo feito em Minas Gerais, que traçou o perfil dos pacientes com AVC cadastrados na estratégia de saúde da família em Diamantina, onde contrastando com achado destes trabalho, $49 \%$ dos indivíduos tinham o hemicorpo esquerdo afetado ${ }^{14,15}$.

A hipertensão arterial (HA) é o principal fator de risco para surgimento de doença cérebro vascular, sua presença aumenta de 3 a 4 vezes o risco de se desenvolver AVC, sendo responsável por pelo menos metade de todos os casos desta patologia ${ }^{16,17}$. Como foi avaliado neste trabalho todos os pacientes tinham HA, sendo as outras patologias associadas, diabetes mellitus (15\%), colesterol (5\%) e arritmias (5\%), tais dados mostram-se semelhantes ao trabalho feito no Hospital Universitário da Universidade de São Paulo, que mostrou as características dos pacientes atendidos neste local, onde mais da metade dos pacientes tinham HA (67\%), seguido de cardiopatias $(24,6 \%)$ e diabetes $(20,4 \%)^{18}$.

A maioria dos participantes da pesquisa utilizavam dispositivo para auxiliar na marcha, o que implica em uma dificuldade em deambular de forma independente, seja pela idade ou pelo próprio comprometimento motor causado pela patologia. Em um trabalho sobre assimetria e desempenho funcional em hemiplégicos crônicos antes e após programa de treinamento em academia, 50\% dos indivíduos faziam uso de órtese e/ou dispositivo para auxiliar na marcha, mostrando desta forma o impacto 
causado pelo AVC na independência funcional dos indivíduos ${ }^{19}$.

$\mathrm{Na}$ avaliação da função motora dos pacientes, foi notado que $70 \%$ tinham restrição de mobilidade articular, sendo esta amplitude diminuída principalmente na flexão e abdução de ombro, supinação de antebraço e na flexão dorsal de tornozelo. Cacho, avaliando a recuperação motora de pacientes hemiplégicos através do protocolo de Fugl-meyer observou que após seis meses de tratamento $20 \%$ dos pacientes ainda tinham limitação de amplitude articular e dor principalmente a flexão, rotação externa e abdução de ombro, seguida de extensão de punhos e dedos, ele atribui este comprometimento a presença de dores no ombro, como principal causa para este fato ${ }^{20}$.

O déficit somatosensorial é um dos mais frequentes resultados da lesão cerebral, nesta pesquisa $70 \%$ dos participantes apresentavam comprometimento sensorial, no que diz respeitos as funçóes exteroceptivas de membro superior, palma da mão, coxa, sola do pé e proprioceptivas dos membros, segundo avaliação sensitiva do protocolo de Fugl-Meyer. As consequências mais evidentes do déficit somatosensitivo são o déficit de reconhecimento tátil e manipulação de objetos, comprometimento do controle motor no membro afetado e déficit no controle do nível de força da mão durante a preensão ${ }^{21}$.

O Acidente Vascular Cerebral frequentemente ocasiona alteraçóes de equilíbrio, decorrentes de lesóes no sistema nervoso central que afetam os aspectos motores, sensoriais e de integração do controle do movimento. Quanto ao equilíbrio, nenhum dos pacientes apresentou normal e $15 \%$ estavam ausentes. Correlação negativa significante entre as pontuaçôes do equilíbrio, sensibilidade e movimentação voluntária do membro inferior do protocolo de Desempenho Físico de Fugl- Meyer. A avaliação do equilíbrio, no entanto, depende de um controle complexo, por envolver o sistema somatosensorial (proprioceptivo, cutâneo, articular), visual e vestibular ${ }^{22}$.

Quando avaliado a função motora dos pacientes, no membro superior $85 \%$ apresentavam alguma alteração e em $15 \%$ a função do membro estava ausente, sendo que as tarefas de movimentação em abdução, adução e retração de ombro foram as mais comprometidas. Foi observado que cerca de $65 \%$ dos sobreviventes de AVC tem limitaçóes funcionais de membro superior ${ }^{23}$, e a recuperação da função de extremidade superior de mais da metade dos pacientes após AVC grave está associada a compensação do membro afetado ${ }^{24}$. A diminuição da função do braço hemiplégico implica dificuldade de realizaçâo das atividades da vida diária e está associada à redução de qualidade de vida ${ }^{25}$.

Alguns autores colocam que a recuperação motora é maior na extremidade inferior em relação ao tempo e qualidade ${ }^{26}$. Nesse estudo função do membro inferior mostrou-se menos comprometida em relação ao superior, onde apesar das funçôes de todos os pacientes estarem comprometidas, em nenhum esteve a função ausente; foi visto também que a dorsiflexão foi a tarefa menos realizadas por eles. No entanto, não foi observada diferença de padrão de recuperação para extremidades superior e inferior, e que a constatação dos autores citados anteriormente deve-se à utilização de medidas de incapacidade física, em lugar de testes específicos para o comprometimento motor das extremidades superior e inferior, já que a função da extremidade superior requer um controle motor fino, que não pode ser comparado com o do membro inferior ${ }^{27}$.

Também foi avaliado o nível de comprometimento motor dos pacientes, sendo visto que a maioria, segundo classificação de comprometimento motor de Fugl-Meyer era marcante $(65 \%)$ e $35 \%$ dos pacientes tinham um comprometimento motor grave. Não sendo encontrada associação entre a idade e o nível de comprometimento motor dos pacientes, em estudo sobre a idade e resultados funcionais após a reabilitaçâo de pacientes com Acidente Vascular cerebral, que mostrou que embora tenha havido um aumento estatístico quando associado entre idade e resultado funcional, não houve uma variação significativa para se explicar que a idade tenha uma relevância clínica sobre a recuperação funcional ${ }^{28}$. No entanto, vale ressaltar que alguns fatores de risco associados a idade, tais como co-morbidades, incluindo doenças cardíacas, hipertensão, diabetes e alterações na capacidade cognitiva, tem influência negativa sobre a recuperação funcional de pacientes idosos, o que explica a associaçáo de alguns autores entre idade e prognóstico negativo de reabilitação.

O tempo de tratamento não interferiu no nível de comprometimento motor dos pacientes, em artigo de re- 
visão de literatura foi discutido os fatores que afetam o resultado funcional de pacientes com Acidente Vascular Cerebral, dentre eles a intensidade da reabilitação, concluindo que quanto mais intenso o tratamento maior a recuperação dos pacientes, não foi discutido sobre o tempo de tratamento como neste trabalho e também não foram encontrados artigos com esta correlaçáo ${ }^{29}$.

\section{CONCLUSÃO}

Este estudo avaliou que a função de pacientes com Acidente Vascular Cerebral em tratamento fisioterapêutico e possibilitou perceber que náo houve associação entre a gravidade do comprometimento motor com a idade e com o tempo de tratamento desses indivíduos.

Existem poucos estudos correlacionando o tempo de tratamento com a função sensório-motora de pacientes tratados com AVC, sugere-se desta forma mais estudos que possam ampliar esta pesquisa, com uma amostra maior para que se possa inferir quais os fatores estão associados à melhora funcional do sistema neuromuscular desses pacientes.

\section{REFERÊNCIAS}

1.Chaves M. Acidente Vascular Encefálico: conceituação e fatores de riscos. Rev Bras Hipertens 2000;7:372-82.

2.Acidente Vascular Cerebral. Informações sobre Doenças Tratadas (endereço na internet). Brasilia: Rede SARAH de Hospitais de reabilitação. (atualizado em: nâo informado; acessado em: 11/2009). Disponível em: http://www.sarah.br/ 3.Zétola VHF, Nóvak EM, Camargo CHF, Carraro JH, Coral P, Muzzio JA, et al. Acidentes vascular cerebral em pacientes jovens análise de 164 casos. Arq Neuropsiquiart 2001;59(3-b):740-5.

4.Lotufo PA, Bensenor IM. Stroke mortality in São Paulo (1997-2003) A description using the Tenth Revision of the International Classification of Diseases. Arq Neuropsiquiatr 2004;62:1008-11.

http://dx.doi.org/10.1590/S0004-282X2004000600014

5.Azeredo JR. Acidente Vascular Cerebral (endereço na internet). Rio de Janeiro. (atualizado em: 01/2009; acessado em: 04/2009). Disponível em: http:// bibliomed.com.br

6.Silva e Souza SR, Oliveira CA, Mizuta NA, Santos MHMR, Moreira AP, Feitosa AL. Reabilitação funcional para membros superiores pós-acidente vascular encefálico. Rev bras Fisioter 2003;4;195-9.

7.Bohannon RW. Relationship between static strength and various other measures in hemiparetic stroke patients. Int Rehabil Me 1986;8:125-78.

8.Teive H, Zonta M, Kumagai Y. Tratamento da Espasticidade: uma Atualização. Arq Neuropsiquiatr 1998;56:852-8.

http://dx.doi.org/10.1590/S0004-282X1998000500025
9.Shelton FN, Reding MJ. Effect of lesion location on upper limb motor after stroke. Stroke 2001;32:107-12.

http://dx.doi.org/10.1161/01.STR.32.1.107

10.Rodrigues JE, Sá MS, Alouche SR. Perfil dos pacientes acometidos por AVE tratados na clínica escola de fisioterapia da UMESP. Rev Neurocienc 2004; 12:117-22.

11. Bruno AA, Farias, CA, Iryia GT, Masiero D. Perfil dos Pacientes Atendidos no Lar Escola São Francisco: Centro de Reabilitação. Acta Fisiátrica 2000;7:92-4.

12.Rolak AL. Segredos em neurologia. 2. ed. Porto Alegre: Artmed, 2001, 480p. 13.Falcão IV, Carvalho EMF, Lessa FJD, Leite VMM. Acidente Vascular Cerebral Precoce: Implicação para o adulto em idade produtiva Atendidos no sistema Único de Saúde. Rev Bras Matern Infantil 2004;4:95-102.

14.Leite H, Nunes APN, Correia CL. Perfil epidemiológico de pacientes acometidos por acidente vascular encefálico cadastrados na Estratégia de Saúde da Família em Diamantina, MG. Fisioter Pesq 2009;16:34-9.

15.Vôos MC, Le RV. Estudo comparativo entre a relação do hemisfério acometido no acidente vascular encefálico e evolução funcional em indivíduos destros. Rev Bras Fisioter 2008;12113-20.

16.Chaves MLF. Acidente Vascular Encefálico: concetuação e fatores de riscos. Rev Bras Hipertens 2007:372-82.

17.Fuchs SC, Lessa JR, Nunes AH. Hipertensão arterial sistêmica e acidente vascular encefálico: a magnitude do risco. Rev Bras Hipertens 2000;7:347-50. 18. Mazzola D, Polese JC, Shuster RC, Oliveira SG. Perfil dos pacientes acometidos por acidente vascular encefálico atendidos na clinica de fisioterapia neurológica na cidade de Passo Fundo. RBPS. 2007; 20(1):22-7.

http://dx.doi.org/10.5020/18061230.2007.p22

19. Teixeira-Salmela LF, Lima RCM, Lima LAO, Morais SG, Goulart F. Assimetria e desempenho funcional em Hemiplégicos crônicos antes e após programa de treinamento em academia. Rev bras fisioter 2005;9:227-33.

20.Cacho EWA, Melo FRLV, Oliveira R. Avaliação da recuperação motora de pacientes hemiplégicos através do protocolo de desempenho físico Fugl-Meyer. Rev Neurocienc 2004;12:94-101.

21.Smania N, Montagnana B, Faccioli S. Rehabilitation of somatic and related deficit of motor control in patients with pure sensory stroke. Arch Phys Med Rehab 2003;84:1692-702.

http://dx.doi.org/10.1053/S0003-9993(03)00277-6

22.Di Fábio RP, Badke MB. Relationship of sensory organization to balance function in patients with hemiplegia. Phys Ther 1990;70:20-6.

23.Dobkin B. Clinical practice. Rehabilitation after stroke. N Engl J Med 2005;352:1677-84.

http://dx.doi.org/10.1056/NEJMcp043511

24.Nakayama H, Jorgensen HS, Raaschou HO, Olsen TS. Compensation in recovery of upper extremity function after stroke: the Copenhagen Stroke Study. Arch Phys Med Rehabil 1994;75:852-7.

http://dx.doi.org/10.1016/0003-9993(94)90161-9

http://dx.doi.org/10.1016/0003-9993(94)90108-2

25.Duncan PW, Wallace D, Lai SM, Johnson D, Embretson S. The Stroke Impact Scale Version 2.0 Evaluation of Reliability, Validity, and Sensitivity to Change. Stroke 1999;30:2131-40.

http://dx.doi.org/10.1161/01.STR.30.10.2131

26.Dombovy ML. Rehabilitation and the course of recovery after stroke. In: Whisnant J P, editor. Stroke: populations, cohorts and clinical trials. Oxford, England, Butterworth-Heinemann, 1993, p.218-37.

27.Duncan PW, Propst M, Nelson SG. Reliability of the Fugl-Meyer assement of sensoriomotor recovery following cerebrovascular accident. Phys Ther 1983;63:1606-10.

28.Bagg S, Pombo AP, Hopman W. Effect of Age on Functional Outcomes 
After Stroke Rehabilitation. Stroke 2002;33:179-85.

http://dx.doi.org/10.1161/hs0102.101224

29.Cufi DX, Stewart DG. Factors Affecting Functional Outcome After Stroke:
A Critical Review of Rehabilitation Interventions. Arch Phys Med Rehabi 1999;80(5 Suppl 1):S35-9.

http://dx.doi.org/10.1016/S0003-9993(99)90101-6 